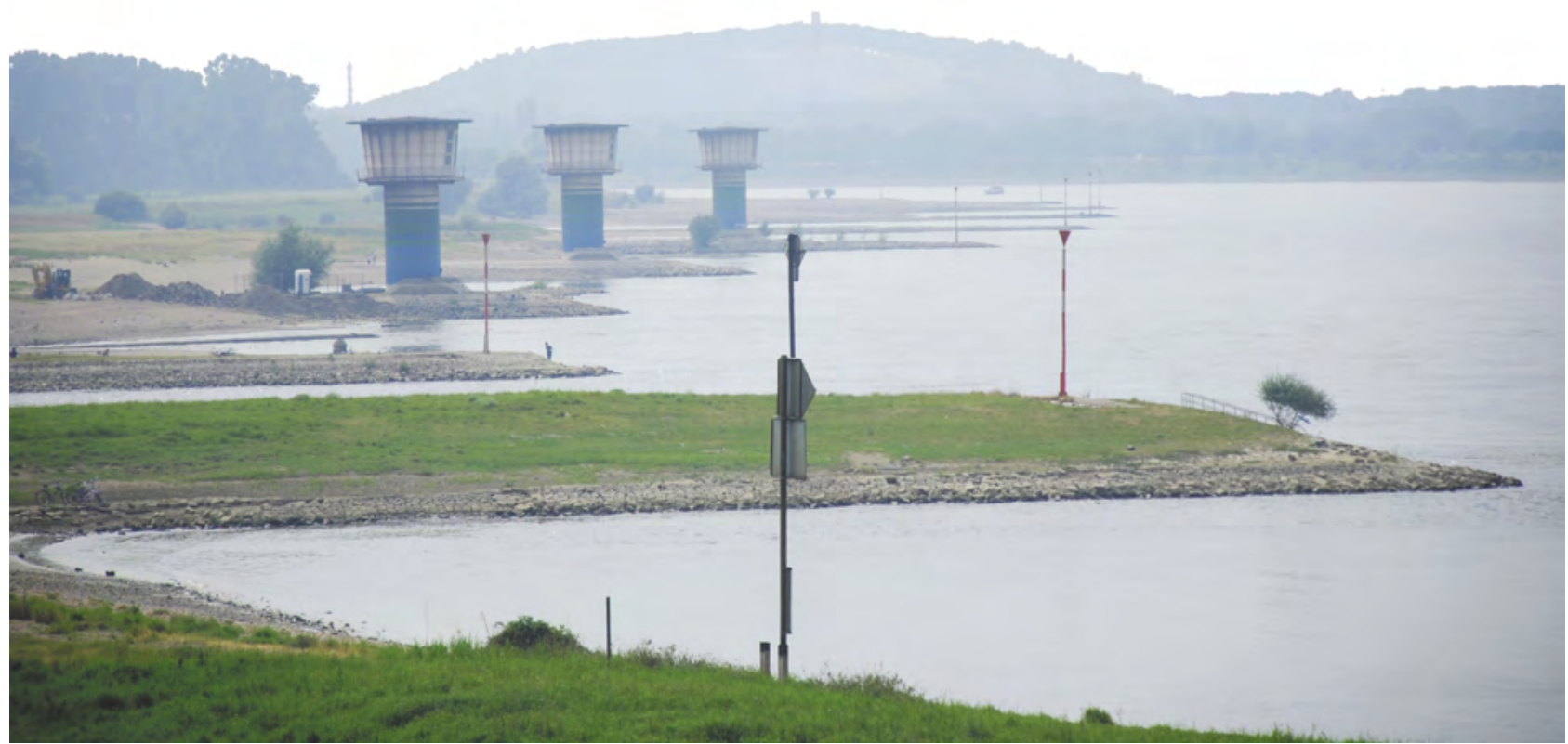

Faculté d'architecture, d'ingénierie architecturale, d'urbanisme de l'Université catholique de Louvain 


\title{
Analyse de références architecturales en Flandres
}

\author{
Marie-Clotilde Roose (dir.), Antoine Ghestem, Constance Leduc
}

\section{Allumer l'œil critique}

L'œil se forme au contact des modèles. Zoom sur le séminaire de "Critique architecturale contemporaine".

\section{Un triptyque}

Parmi les trois séminaires de recherche critique à Tournai en appui aux TFE1, le séminaire de critique architecturale contemporaine comporte trois volets, tenus par trois enseignants. Jérôme de Alzua, architecte praticien, organise le choix des visites et supervise les études de cas. En 2016, le séminaire fut centré sur Cologne ${ }^{2}$; en 2017, sur la Flandre belge. Chercheur HDR en architecture, Frank Vermandel a initié l'étude critique et la comparaison de revues européennes, telles que Faces (Suisse), $\mathrm{A}+$ (Belgique), D'A (France), Le Visiteur, Criticat... Le $3^{e}$ volet, proposé par Marie-Clotilde Roose, se situe au niveau philosophique, éveillant les étudiants à l'épistémologie, à ses ouvertures vers la phénoménologie, aux implications du terme critique. Les objectifs généraux du séminaire étant de mener un travail de recherche engagé où développer l'esprit critique, porter un regard avisé sur une question d'actualité, construire une critique architecturale sur base d'un argumentaire valide, nourri de références.

\section{Processus}

Un $1^{\text {er }}$ tour de table a permis de lister les thèmes de TFE, écouter les attentes concernant ce séminaire, et en présenter les étapes. Une liste de bâtiments contemporains remarquables a été proposée ; chaque étudiant(e) a pu en choisir un, si possible en lien avec ses recherches de TFE, et a formé un binôme.

À partir de l'article "Connaissance » de l'Encyclopædia Universalis, un débat a pris place, confrontant théories et expériences des étudiants. Comment se construit et se partage une connaissance ? Quelle en est la part dite objective, et la part subjective dans l'interprétation des observations ? À quoi se rendre attentif, dès lors qu'on touche à des questions de sens et de valeurs? Quels retours possibles sur les Critiques de Kant, vues en baccalauréat: Que puis-je savoir ? Comment agir ? Qu'est-ce que juger ? Enfin, l'épistémologie s'ouvrant à la phénoménologie dès le $x x^{e}$ siècle, l'article « Une pratique concrète ${ }^{3}$ de Natalie Depraz a permis de saisir les clés de la méthode à suivre pour... la visite.

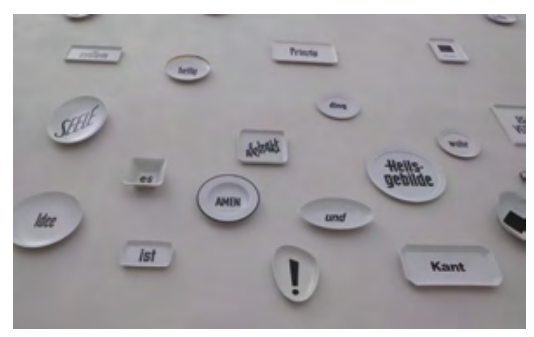

Munis de ces consignes de description phénoménologique (sans recherche préalable), les étudiants se sont rendus en solo ou duo sur les 26 lieux à découvrir en autonomie, avec appareil photo et carnet de notes. Pour rendre par écrit cette description naïve du parcours vécu. Ensuite, ils ont commencé à se documenter (plans, coupes, façades...) et à chercher des articles à propos de ces bâtiments - ou similaires, car il n'a pas toujours été possible d'en trouver (même en néerlandais).

Ensuite, la comparaison de revues a pu commencer, précédée par un exposé théorique. Comment une revue se distingue-t-elle d'une autre, au niveau du contenu et de la forme? Quels sont les éléments à repérer, comme l'éditorial, le comité de lecture, le public visé... ? Comment l'article se structure-t-il ? Quelles en sont les qualités d'écriture? Le rédacteur s'engage-t-il dans une conclusion personnelle ? Le choix des illustrations et de la mise en page est-il pertinent et révélateur? En binômes, les étudiants ont dû présenter à tous l'étude comparée de leurs cas et articles, évalués en fonction de la qualité des analyses sur les bâtiments étudiés.

Enfin, les étudiants ont préparé un dossier final, reprenant de manière critique les découvertes faites en utilisant ces outils, ces trois volets à partir desquels peindre leur propre paysage. Qu'en ontils retenu, et comment les utiliser pour leur TFE ? Quelles difficultés à surmonter ? Quelles étapes franchies, avec quelles satisfactions ou réserves? Ceci, en auditoire ouvert, afin de stimuler la réflexion collective.

\section{Des résultats motivants}

Les dossiers furent, pour la plupart, soignés et bien étoffés, avec des critiques architecturales de belle facture. Nombre de ces bâtiments en Flandre, neufs ou rénovés, sont récents. Les proposer à Lieuxdits offre donc un triple intérêt : une émulation pour les étudiants, un reflet du travail pédagogique, et des critiques inédites home made \& made in Belgium.

Parmi ces lieux dits par de futurs architectes, en voici un diptyque.
1 Photo réalisée par M.-C. Roose au musée Kolumba, 14 nov. 2016.

Cadrée sur l'installation d'Anna Blume (1936-), à partir de" 71 Designs for Editions in Porcelain" (1985) par Bernard J. Blume (1937-2011)

1 - Travail de Fin d'Études.

2 - Roose M.-C., « Parcours phénoménologique de la Chapelle et du Kolumba de Zumthor », Lieuxdits $\mathrm{n}^{\circ} 12$, mai 2107 ,pp. 14-17.

3 - Le dossier « La phénoménologie aujourd' hui », Le Magazine littéraire $n^{\circ} 403$, Nov. 2001, pp. 19-28. 


\section{L'entrepôt St. Felix, le rejet du conformisme des programmes}

\author{
Antoine Ghestem
}

L'entrepôt St. Felix est un exemple impressionnant sur le plan architectural d'un entrepôt du xix ${ }^{e}$ siècle. Le bâtiment est construit en 1860 comme lieu de stockage de marchandises en vrac comme le café, le grain, le fromage et le tabac. En 2006, le lieu est transformé par l'agence Robbrecht en Daem, pour pouvoir y stocker les archives de la ville, ainsi que celles de l'autorité portuaire. En tout l'ancien entrepôt contient désormais entre ses murs $24 \mathrm{~km}$ d'archives, dont des documents inestimables datant du $x v^{e}{ }^{e}$ siècle. Mais ce bâtiment est bien plus qu'un entrepôt d'archives.

\section{Un lieu}

En premier lieu, la caractéristique la plus importante de ce bâtiment est son emplacement. En effet, la ville d'Anvers fait partie de ces villes dynamiques qui investissent pleinement dans la question des espaces publics notamment la réaffectation des espaces industriels en cœur de ville comme étant des lieux clefs pour apporter une plus-value à la ville. Ainsi Anvers " consacre depuis 2007 le tiers de son budget au développement urbain $»$. Le quartier du port historique, Eilandje, fait partie avec le parc Spoor Noord d'une des réaffectations phare de ce programme. Or l'entrepôt de St. Félix est au cœur du port historique et de son nouvel aménagement, et possède la particularité d'être composé d'une véritable rue intérieure, reliant les quais du port au boulevard Oude Leeuwenrui. Là où jadis, les marchands du port stocker leurs marchandises, la commune d'Anvers va en faire une porosité urbaine conjuguée à une fonction, un lien entre les quartiers. Une de ces petites interventions qui permettent, l'une après l'autre, de rendre la ville parcourable à pied et agréable. Surprenante.

\section{Des programmes}

Car en effet, la deuxième caractéristique importante de ce projet, est l'offre programmatique. Ordinairement, un lieu au potentiel de $22000 \mathrm{~m}^{2}$ en centre-ville, dans un des quartiers en passe de devenir l'un des quartiers les plus attractifs de la ville, aurait pu recevoir ces lots de programmes habituels. Tel qu'un centre commercial nouvelle formule comme tous ces supermarchés city, couplé avec un cinéma et des restaurants fast-food et autres chaines de cafés standardisées.

Mais loin de là, très loin de là, Anvers décide de placer dans ce lieu stratégique, certainement très appétissant pour les promoteurs, les archives de la ville ainsi que des espaces publics pour leurs consultations. Anvers fait le pari courageux de marier cette nouvelle place devenu tendance et populaire, avec un programme qui pourrait être qualifiée de confidentiel, et encore cela serait un euphémisme. Le choix est puissant. Et pour continuer à donner une image plus attractive aux mémoires de la ville et de ses habitants, la commune décide d'installer au rez-de-chaussée différents restaurants, et cafés branchés. Ainsi, les Anversois prennent désormais leurs brunchs au soleil, sur la terrasse des archives communales face au port historique. L'image est séduisante. Le pari de rendre attractif, sexy et populaire la mémoire et sa connaissance paraît réussi.

\section{Une mise en scène}

Dans ce lieu classé, le projet d'architecture se devait d'être tout en délicatesse. L'intervention contemporaine est ponctuelle. Mais ce lieu, n'était-il pas déjà moderne dans le sens où il propose des espaces capables? Le bureau Robrech en Daem prend le parti de diviser l'entrepôt en étages bien différenciés. Le rez-de-chaussée et la rue intérieure sont alloués à l'accueil du public, tandis que les combles sont réservés à la consultation. Les cinq autres niveaux intermédiaires reçoivent les kilomètres d'archives. Ces niveaux sont invisibles pour le visiteur, l'essentiel étant de préserver les archives de la lumière et de l'humidité.

Phénoménologiquement, l'expérience est étonnante. Le visiteur souhaitan consulter les archives passe devant ou aperçoit des monuments contemporains iconiques, tels que la capitainerie de Zaha Hadid, ou le Museum aan de Stroom de Willem Jan Neutelings et Michiel Riedijk. Puis traverse les terrasses vivantes de l'entrepôt St. Félix, rentre dans la monumentale et bruyante nef lumineuse de l'entrepôt tout immaculée de blanc, cherche l'accueil à travers une des nombreuses anciennes portes de cette rue intérieure, entre dans une cellule aux murs de briques peintes en blanc et prend l'ascenseur. Lorsque les portes s'ouvrent six niveaux plus haut l'atmosphère a radicalement changée. Le son est sourd. L'ambiance est extrêmement calme et studieuse. Dans ce lieu habillé d'ancien parquet, et de lourds rideaux sombres, le contraste avec l'univers que nous venons de quitter est très fort. Suffisant pour permettre au visiteur d'avoir naturellement la bonne atti- 
tude pour ce lieu d'étude. Les volumes sont un paysage de pentes et de sous pentes en lames de chêne, permettant de distribuer les différents espaces de lectures, de loges et de conférences. Dans cet univers, les rares ouvertures sur l'extérieur ne permettent pas une connexion avec le dehors. En effet les seuls puits de lumière ne donnent pas à voir sur l'extérieur. La lumière nous vient des lampes accrochées sous les sous pentes, et des dizaines de lampes de bureaux individuelles. Cette atmosphère close sur elle-même, sobre, composée uniquement par le rythme des toitures et par les très longues tables, où les chercheurs étudient côte à côte, fait penser aux réfectoires monastiques. Un détail est particulièrement révélateur de cette attention de vouloir se raccrocher à ces ambiances ancestrales : les lampes de bureau individuelles s'allument en tirant sur une chainette, telles les anciennes lampes à tirette des illustres bibliothèques. De cette salle de consultation, les archives sont invisibles. Aucun livre. Les archives se commandent, et arrivent individuellement par monte-charge, puis sont distribuées par l'intermédiaire du personnel et sont consultées sur un cousin de présentation. Ces multitudes d'actions rendent l'opération extraordinaire, l'archive précieuse. Ce projet donne l'impression d'un lieu hors du temps, sans localisation particulière, ou l'on pourrait commander infiniment de la connaissance sur coussin, piochée dans une grande fourmilière articulée sur plusieurs niveaux sous nos pieds. Tandis que dehors, contre la façade du bâtiment de la mémoire, la vie bat son plein.

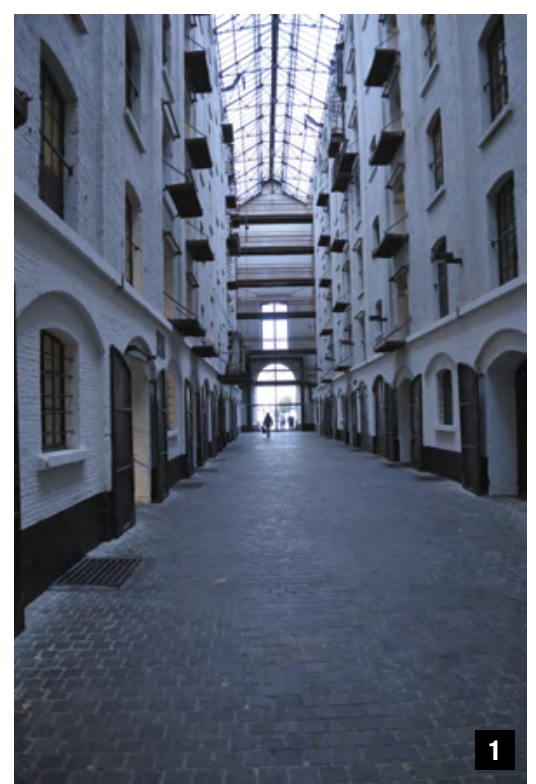

Rue intérieure, photo : Antoine Ghestem

2 Partition autour d'un vide «St-Felix Warehouse Antwerp, Belgium, $2006 »$, www. robbrechtendaem.com, http:// www.robbrechtendaem.com/ projects/libraries-archives/st.-felix-warehouse-antwerp, consulté le $13 / 01 / 2018$ 


\section{Le Centre du Zwin : un bâtiment fort de ses enjeux paysagers?}

\author{
Constance Leduc
}

\section{Des atouts naturels uniques.}

Au nord-ouest de la Belgique, la Province de Flandre-Occidentale est une région aux caractéristiques qui la rendent unique. À la fois voisine directe de la France et de la Hollande, sa limite supérieure est, quant à elle, la côte de la mer du Nord. Cette disposition avantageuse permet à la Flandre-Occidentale de bénéficier d'un paysage remarquable composé de dunes donc, mais également de plaines humides et de polders. Au nord-est de cette Province se trouve la ville de Knokke-Heist. Si cette commune de 35000 habitants est aussi populaire et reconnue aujourd'hui comme la station balnéaire chic de la côte belge, c'est qu'elle peut compter sur ses indéniables atouts paysagers. Non seulement la ville profite d'une plage de $12 \mathrm{~km}$ de long, mais elle abrite aussi une réserve naturelle dont la réputation dépasse les frontières du plat pays : le Zwin.

Situé à quelques centaines de mètres seulement de la frontière hollandaise, c'est en s'intéressant à la signification de son appellation que l'on comprend mieux l'histoire de sa formation. En effet, le mot Zwin vient d'un mot germanique désignant "une lagune parallèle au rivage marin, comme ces mares retrouvées sur l'estran à marée basse"1. Par ailleurs, "si nous remontons les siècles, au cinquième, se produisit, ce que l'on appellerait aujourd'hui "une inondation catastrophique", qui pénétra de 25 kilomètres à l'intérieur des terres, en détruisant tout sur son passage"2. $\mathrm{Ce}$ bouleversement naturel provoqua la fragmentation du littoral et créa "des chapelets d'îles, des goulets, des chenaux et un bras de mer."2

Le Comte Léon Lippens créa la réserve naturelle en 1952. À partir de 1990, la plaine du Zwin et ses infrastructures pédagogiques furent réaménagées et modernisées pour devenir en 2016, le Zwin Parc Nature. C'est dans ce contexte que le bâtiment du Centre du Zwin voit le jour la même année. Aujourd'hui, la réserve naturelle du Zwin s'étend sur une superficie de 150 hectares, dont 25 aux Pays-Bas. Cette mise en situation nous permet de comprendre les tenants et les aboutissants du projet d'architecture qui en découle.

\section{L'humilité de l'approche}

Ce sont les architectes gantois Ralf Coussée et Klaas Goris qui, en 2012, remportent le concours organisé par la Province de Flandre-Occidentale pour la réalisation du nouveau centre des visiteurs et l'aménagement de la réserve. Diplômés en 1983 et 1984 de l'Académie d'Architecture Saint Lucas à Gand, les deux architectes ont enchaîné quelques collaborations avant de finalement se retrouver pour fonder le bureau Coussée \& Goris Architecten en 1998. Ils sont à l'origine de plusieurs projets reconnus pour leur travail structurel rigoureux, leur humilité et la poésie qui s'en émane, tels que le crématorium Hofheide à Holsbeek ou encore la bibliothèque Waalse Krook à Gand.

L'approche vers le Centre des visiteurs du Zwin est étonnante par la couleur qu'il revêt. En effet, le bâtiment en pin Douglas est noir, presque comme s'il avait brûlé ; un choix de non-couleur audacieux que certains trouveront austère mais qui finalement se révèle astucieux dans son rapport au paysage. Cette teinte sombre offre au bâtiment une certaine humilité puisqu'il permet de créer un contraste important avec la douceur des couleurs du paysage et ainsi, de les mettre en valeur.

Après avoir rejoint le grand parking paysager destiné à accueillir voitures et autocars de tourisme, marcher vers le Centre d'accueil nous amène dans ce qui se veut être une cour centrale (voir zone beige sur le plan Figure 1), formée par la disposition en plan de quatre bâtiments. Cependant, rien, à part un long banc en bois noir, ne prédestine cet espace à devenir un vrai lieu de rencontres. Cette "cour centrale" est en fait plus un lieu de passage qui mène tout droit à l'entrée du Centre. Le sol minéral marque clairement l'arrivée dans ce lieu architecturé qu'est le Centre des visiteurs, point de rassemblement de la réserve.

\section{Un programme engagé ?}

Le Centre d'accueil des visiteurs se développe sur deux niveaux. Au rezde-chaussée, il regroupe entre autres une billetterie, un espace d'exposition permanente, une boutique et un espace de restauration. Ce grand hall, d'une taille certes questionnable, exerce cependant une vraie fonction de pivot entre ces différents programmes. C'est également depuis ce bâtiment que se trouvent les accès à la réserve naturelle. Dans l'espace d'exposition permanente sur les oiseaux migrateurs, la structure, pensée par l'ingénieur Guy Mouton, est particulièrement efficace quand il s'agit d'étendre ses fonctions de contreventement à la création de sous-espaces inté- 
rieurs, destinés à guider le visiteur dans sa visite. La disposition en sinusoïde des poutres obliques crée donc une sorte d'ondulation dans la promenade intérieure que propose ce long bâtiment de $135 \mathrm{~m}$, qui sans elle, aurait pu paraître quelque peu monotone.

Au niveau supérieur, on retrouve un espace d'exposition temporaire et un accès à la "tour" d'observation, qui se veut être un signal depuis la piste cyclable qui longe le bâtiment. Cette tour d'observation a été voulue par les architectes pour montrer qu'il y a quelque chose d'intéressant à voir pour les promeneurs qui s'approchent de la réserve, et également permettre d'avoir une vue d'ensemble une fois là-haut. Cependant en pratique, la hauteur de la tour n'est absolument pas assez conséquente pour créer un signal dans le paysage. De plus, une tour renvoie à l'existence d'un point haut vertical, ce qui n'est absolument pas le cas ici (cf. figure 1), où rien ne dépasse de la ligne d'horizon. Cette tour d'observation aurait pu être un vrai landmark si elle avait été assumée jusqu'au bout, visible, imaginons-le, depuis la plage de Knokke-Heist par exemple. En haut de cette tour, on perçoit très légèrement la mer, mais la hauteur n'est pas suffisante pour comprendre exactement comment s'est formé le Zwin. Les étangs, les dunes, les chapelets d'îles, les goulets, les chenaux, le bras de mer... dans un cas comme celui-ci, l'architecture doit saisir pleinement les moyens qu'elle a en sa possession pour faire naître chez le visiteur l'envie de comprendre, comprendre comment sa région s'est formée, quels sont les évènements naturels à l'origine du paysage qu'il a sous les yeux aujourd'hui. Malheureusement, le bâtiment ne permet pas de voir beaucoup plus que ce que la promenade au sol ne nous offre déjà.

En conclusion, face à un tel paysage, les architectes Coussée et Goris ont su répondre avec humilité à l'implantation du bâtiment dans son environnement, sa couleur noire mettant magnifiquement les douces tonalités du paysage en valeur, mais ont peut être mis une énergie considérable dans le fonctionnement du bâtiment en lui-même, avec sa cour et son hall d'accueil gigantesque, plutôt que dans ce que le bâtiment permet d'offrir comme regard sur le paysage. D'un environnement comme celui-ci émanent des enjeux pédagogiques, politiques et poétiques, à saisir absolument au vu des impératifs écologiques auxquels nous sommes confrontés aujourd'hui.

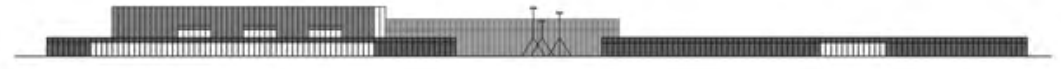

Pas de rupture verticale visible en élévation malgrél'existence d'une «tour» d'observation

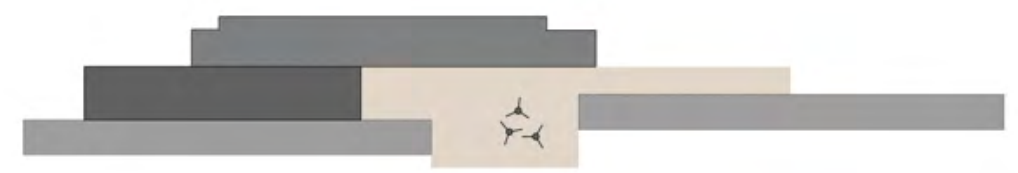

La cour, lieu de rencontres ou lieu de passage?

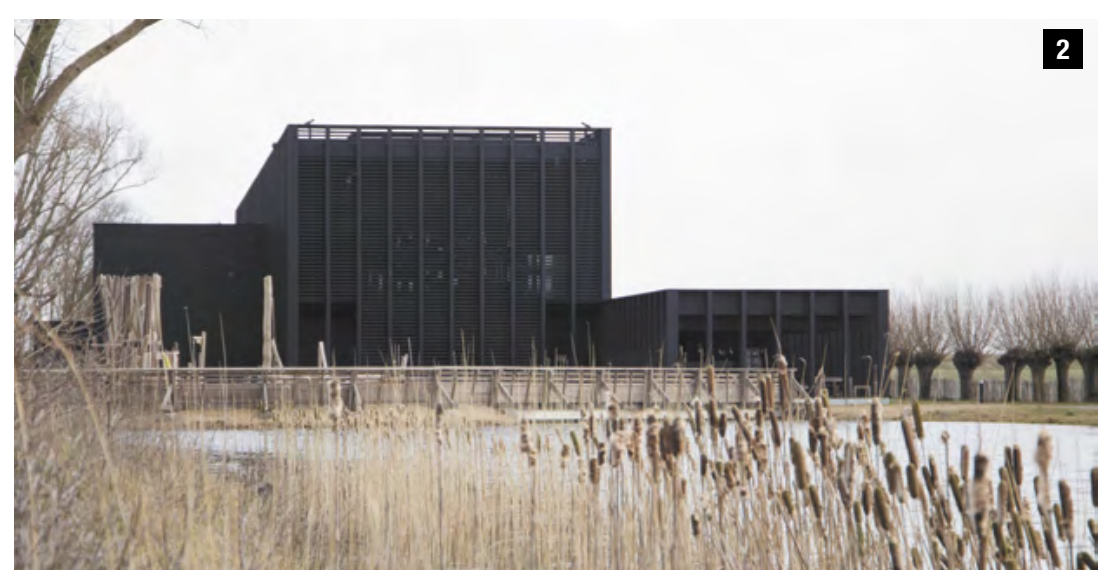

Elévation et plan du Centre du Zwin, image libre de droit Crédit : Constance Leduc image libre de droit Crédit : Alexandre Olivier, étudiant M1 UCL/LOCITournai

La non-couleur noire du bâtiment se met au service des teintes naturelles du paysage 

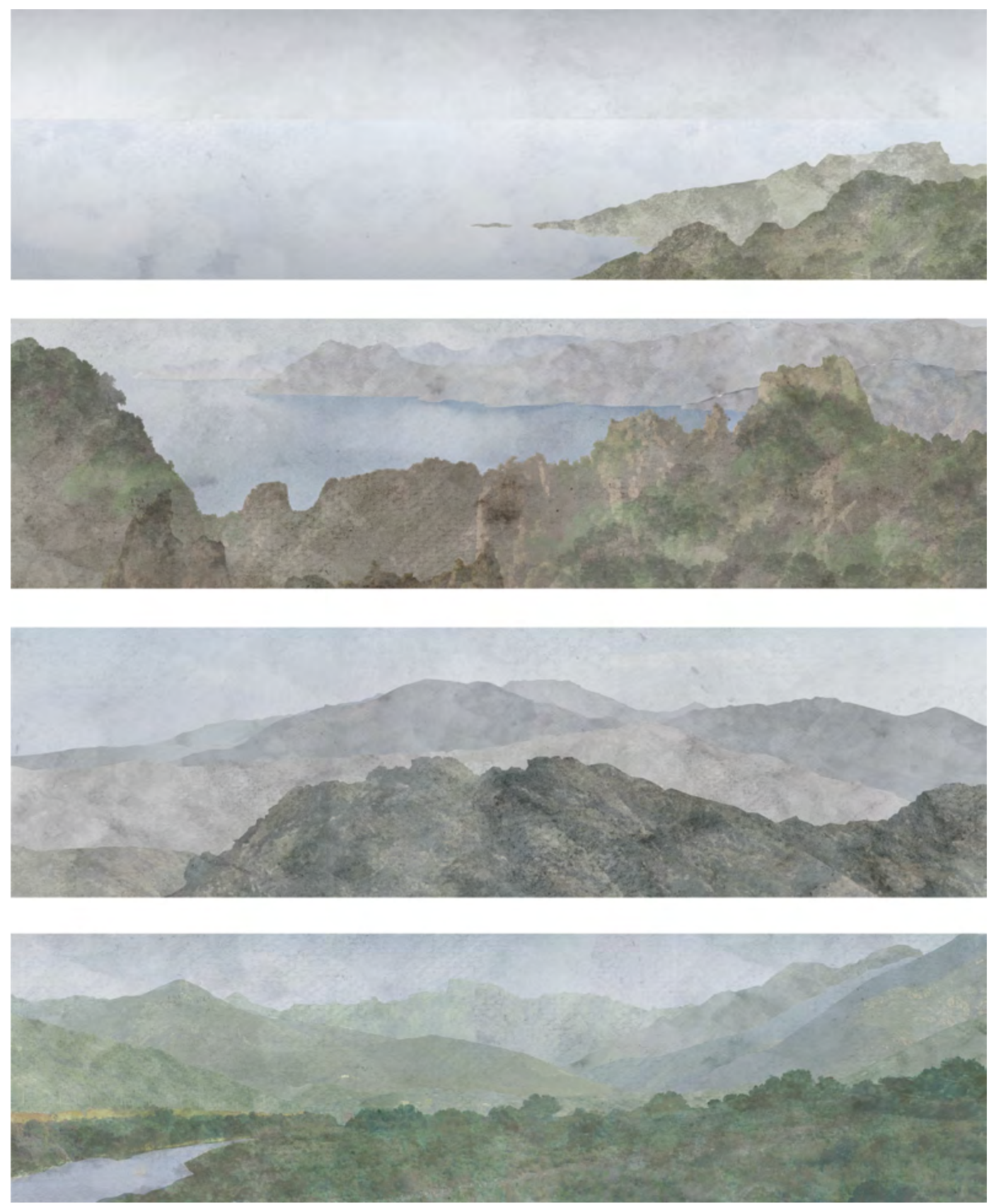